\title{
Note sur le sacrifice chez les Arabes musulmans
}

A note on sacrifice among Arabian Muslims

\section{Nicole Grandin}

\section{OpenEdition}

\section{Journals}

Édition électronique

URL : http://journals.openedition.org/span/371

DOI : 10.4000/span.371

ISSN : 2268-1558

\section{Éditeur}

École pratique des hautes études. Sciences humaines

\section{Édition imprimée}

Date de publication : 1 septembre 1978

Pagination : 87-114

ISSN : 0294-7080

\section{Référence électronique}

Nicole Grandin « Note sur le sacrifice chez les Arabes musulmans », Systèmes de pensée en Afrique noire [En ligne], 3 | 1978, mis en ligne le 04 juin 2013, consulté le 19 avril 2019. URL : http:// journals.openedition.org/span/371 ; DOI : 10.4000/span.371 


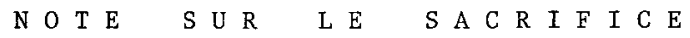

C HEZ LES ARABES M U S U L M A S

par Nicole Grandin

Les Arabes ont toujours pratiqué le sacrifice sanglant, dans $1^{\prime}$ antéislam comme aujourd'hui. Mais le passage des tribus du Hêjaz, au VIIe siècle, du polythéisme au monothéisme avec Mahomet, tandis que se conservait à la foị dans et hors de l'islam la pratique du sacrifice, oblige à situer sur trois plans l'étude du sacrifice dans ces sociétés. tel qu'il se présente actuellement.

1) Le plan de 1'islam officie1, dit orthodoxe, auquel appartiennent les sacrifices dits religieux, qui ont lieu au moment du pèlerinage à la Mekke (10 dhul'hijja). Ces sacrifices sont effectués soit à la Mekke même, au cours du pèlerinage, soit ailleurs dans le monde musulman, à la même période. Ils sont mentionnês dans le Coran et dans la Tradition et s'adressent directement à Allah.

2) Le plan de $1^{\prime}$ islam populaire qui comprend les multiples sacrifices que pratiquent effectivement les populations arabes musulmanes, en diverses occasions et à diverses fins. Ces sacrifices, qui ne sont pas mentionnés dans le Coran et la Tradition, et sont même condamnés par ces textes, s'adressent soit directement à Dieu, par l'entremise d'un saint, soit à un mort, à un ancêtre ou même à un jînn.

3) Enfin le plan de l'anté-islam qui constitue la souche commune dont sont issus ces deux ordres de sacrifices, le premier, celui de $l^{\prime}$ islam officiel, venu, selon les docteurs eux-mêmes, de la reprise par Mahomet du sacrifice anté-islamique pratiqué à la Mekke, les seconds se situant vraisemblablement dans la continuité pure et simple de multiples pratiques liées aux cultes de $1^{\prime}$ anté-islam.

La rareté et la fragilité des données que l'on possède sur ce 
dernier plan rendent déjà la recherche difficile, mais d'autres êlêments viennent la rendre encore plus complexe :

-- Tout d'abord le fait que les sociétés arabes sont des sociétês historiques (on entendra par 1à des sociétés dont l'histoire est connue et s'articule sur I'histoire de l'ensemble de la région avant et au moment de la prédication de Mahomet). Ceci implique, en particulier, de prendre en compte d'une part des données telles que le fond sémitique ancien et surtout $1^{\prime}$ influence sur 1 'islam naissant du judaîsme

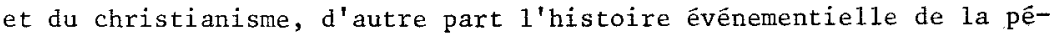
riode de la prêdication.

-- En second lieu, l'existence dans ces sociétés, et dès les premiers temps de l'islam, d'une pensée spéculative, réfléchie, animée du souci de justifier, par la construction d'un modèle dogmatique, le système social et religieux.

-- Enfin, un aspect de l'islam qui a été maintes fois souligné, c'est qu'il se veut à la fois religion et communauté temporelle, c'està-dire qu'il prend en charge, en une même démarche, les relations de chaque croyant avec Allah et les relations des croyants les uns avec les autres, sur le plan moral, social et politique. Les prescriptions coraniques, qui sont considérées en bloc comme rêvélẻes, portent donc aussi bien sur le spirituel que sur les modalités du culte, personnel et communautaire (prière, aumône légale, jeûne du Ramadan, pèlerinage à la Mekke) et le code de vie sociale. Cette démarche unique, qu'il s'agisse du religieux ou du temporel, se reflète profondément, on le verra, sur la manière dont les docteurs ont fixé, commentë ou interprété les rites sacrificiels, et interdit dans le cours de la recherche, toute separation arbitraire entre le domaine religieux et le domaine social.

L'ampleur du champ à saisir pour une étude approfondie est évidente. Dans ces notes, on se limitera volontairement à un bref aperçu phénoménologique du sacrifice "religieux" et à quelques indications concernant le sacrifice populaire dans ses formes les plus courantes. On n'abordera, par ailleurs, que le domaine de l'islam sunnite -- largement majoritaire au Proche-Orient comme en Afrique. En ce qui concerne le sacrifice religieux, on ne retiendra donc que les interprétations des quatre grandes écoles de jurisprudence religieuse ( $f i q h$ ) qui se constituèrent au IIIe siècle de 1'Hégire (début de la période abbaside) et 
qui se partagent aujourd'hui toute 1'étendue de l'islam sunnite.

La plus ancienne, l'école malékite (du non de son fondateur, le juriste Malik ibn Anas, m. en 795) est de loin la plus suivie en Afrique noire et au Maghreb. Née à Médine, 1'école malékite admet comme source du fiqh, outre le Coran et la Sunna, le consensus, ijma, des docteurs et le jugement personnel, ra'y, de chacun d'eux. En outre, elle accorde une place relativement importante à une source généralement considérée comme secondaire, le 'urf, ou coutume. C'est ainsi que le malékisme, en particulier au Maghreb, s'est exprimé dans une jurisprudence où les pratiques locales tiennent une grande place.

L'êcole hanéfite, fondēe en Iraq par le juriste Abu Hanifa (m. en 767) est surtout suivie en Iraq, en Syrie et en Turquie et par les sunnites d'Asie. Elle insiste sur l'utilisation du jugement personnel, ra'y, ce jugement étant finalisé par la recherche du mieux. Elle utilise largement le qiyas, ou raisonnement par analogie, et s'efforce à la recherche du motif, 'izza, qui fonde cette analogie.

L'école shâfi'ite, fonđée par al-Shâfi'i, juriste arabe de la tribu de Quraysh (m. en 820) domine en Basse-Egypte, dans une partie du Héjaz, de 1 'Arabie du Sud, de 1 'Afrique orientale et aussi en Extrême-Orient. Elle valorise la Sunna et y associe le consensus, ijma, mais non plus limité aux docteurs à une êpoque donnẻe, comme le malëkisme et le hanéfisme. Le consensus qu'elle reconnâ̂t est celui de la Communauté.

La quatrième, l'école hanbalite, fondée à partir des recueils du grand traditionniste Ibn Hanbal ( $m$. en 855 ) doit à cette origine des conceptions très différentes des autres écoles : n'admettant que 1a tradition du Prophète et des premiers compagnons, le taqlid, ou acquiescement au passé -- un passé toujours vivant -- est seul admis. Le souci des valeurs morales tend à y primer les solutions juridiques. Ecole de pensée, le hanbalisme déborde les cadres proprement juridiques et connut à plusieurs reprises une grande faveur dans les milieux populaires. Aujourd'hui, le hanbalisme est l'école officielle en Arabie Séoudite.

Chaque école (madhhab) a pris position sur les différents rites liés au sacrifice religieux et les règle jusque dans le détail, en fonction de la signification qu'elle attribue et, partant, de l'importance qu'elle accorde, à chacun d'eux, dans l'ensemble du rituel ( 1 ). Dans le cadre de cette note, il ne sera pas possible de donner la 
position de chaque école pour tous les éléments du rituel et on se limitera à des exemples.

\section{LES SOURCES}

L'étude du sacrifice -- religieux et populaire -- fait appel à quatre types de sources:

1) La Littérature iszomique. Tout d'abord le Coran. Il est le seul ouvrage authentique qui renseigne sur l'antê-islam, notamment par des mentions de culte d'idoles, de pratiques à proscrire, de réactions du Prophète concernant diverses coutumes, etc. D'autre part, il est le fondement de la jurisprudence musulmane et de toutes les pratiques religieuses. Mais, on le sait, son texte n'a êté fixẻ par êcrit, dans la version officielle connue aujourd'hui, que sous le Calife othman, c'està-dire plus de dix ans après la mort de Mahomet et de nombreux versets sont imprécis ou ambigus, ou encore semblent en contredire d'autres... D'où des exégèses, souvent divergentes, inspirées par des motifs politiques ou autres, suivant les époques. En ce qui concerne les sacrifices religieux, les indications donnêes par le Coran sont limitées aux grandes lignes des principales formes, et on n'y trouve rien sur des points tels que le rituel du sacrifice, la consommation de la viande, etc. En revanche, on y relève la condamnation de certaines pratiques sacrificielles anté-islamiques.

Le had̂th (pl. ahâdith) ou dire du Prophète, dont l'ensemble forme la Sunna ou Tradition, et dont on a tiré la Sîra, ou biographie traditionnelle du Prophète (Ibn Hicham, m. en 834). Ces textes fournissent le même type d'informations que le coran, mais leur "fiabilité" a été

(1). S'il y a divergences de vues entre les écoles (madhăłíb), il n'y a cependant pas opposition. Toutes quatre sont egalement orthodoxes et admises à égalité dans l'islam sunnite. 
remise en cause par la critique historique moderne. En effet, les recueils. de hadîth sont très nombreux (les plus estimës sont les deux sahth, "sains", "authentiques" de Bukhari (m. en 870) et de Muslim (m. en 875)) ils portent sur les actes et paroles du Prophète, mémorisés du vivant de Mahomet par ses compagnons et communiqués oralement, par des chaînes de transmetteurs, aux gênérations suivantes jusqu'au IXe siècle. Beaucoup de had̂̂th ont pu ainsi être fabriqués ou dénaturés par des érudits d'origine diverse, pour des motifs sociaux ou politiques. Une méthode de critique externe a certes été êlaborée par les "sciences du hadžth" (mention de la chaîne des garants, isnâd, prouvant $1^{\prime}$ authenticité du fait rapportê), mais elle n'est en rien à $1^{\prime}$ abri d'une critique historique sêrieuse puisqu'elle ne procède pas à 1 'analyse critique du texte, math, du hadith. Aussi, d'un recueil de hadith à 1 'autre, on se trouve en face de contradictions qui permettent des interprétations souvent opposées.

2) La littérature conté-istamique. On a beaucoup écrit sur l'importance du poète dans la société bédouine antē-islamique : veillant au prestige de son groupe, relatant publiquement ses hauts faits, étendant sa renommée et insistant sur sa puissance, il entretenait ses contribules dans 1 'exaltation guerrière en célébrant leurs victoires dans les rêunions intertribales comme la célèbre foire annuelle d'Ukaz ( 1 au $\left.20 d h u l^{\prime} q a^{\prime} d a\right)$, où des joutes avaient lieu entre poètes. L'authenticité des oeuvres poétiques anté-islamiques connues aujourd'hui n'avait guère êté mise en doute avant la publication, en 1927, de la thèse de Taha Hussein, Fi乙'adab-i乙-jâhili. L'islam classique, en effet, animé de la conviction intime que sa propre perfection se situe à ses origines, avait attribué à ces textes un caractère quasi sacré. Mais Taha Hussein va soutenir qu'ils étaient pour l'essentiel apocryphes, donc à rejeter comme source d'information sur les anciens Arabes. Plusieurs auteurs aujourd'hui, comme Bichr Farès (L'honneur chez les Arabes avant Z'islam, Paris, 1923) et Joseph Chelhod (Le sacrifice chez les Arabes, Paris, 1955) estiment cependant qu'apocryphe ou non, cette poésie a valeur de source pour l'anté-islam, dans la mesure où 1 'on n'y cherche pas la relation de faits historiques mais le reflet d'une conscience collective : on sait que le coran a condamné ces poètes de l'antéislam (jâhiliya : ignorance) en des termes vigoureux : "De même les 
poètes sont suivis par les Errants Ne vois-tu pas qu'en chaque vallée ils divaguent et disent ce qu'ils ne font point" (Sourate XXVI, 224226 , trad. Blachēre). Pour l'ethnologue, incontestablement, ces "divagations" mêmes ont valeur de témoignage et ne doivent pas être rejetées.

3) La littérature ethnographique et les récits de voyagé. D'intêrêt inègal, ces textes renseignent surtout sur le sacrifice populaire tel qu'il est pratiqué par les différents groupes arabes. En ce qui concerne le sacrifice religieux à la Mekke, au dêbut đu XIXe siècle, on possède les térnoignages de Burckhardt (Travels...) et de Burton (A pilgrimmage...) qui se mêlèrent aux pélerins. Un dépouillement systématique et comparatif de ce type de sources pour les pratiques sacrificielles populaires dans le monde arabo-musulman reste encore à faire.

4) La phizologie.Certains auteurs, comme Joseph Chelhod (Le sacrifice chez les Arabes, pp. 40-41) lui accordent une place importante dans l'étude de la période anté-islamique et estiment y trouver des matériaux non seulement abondants, mais d'une sûreté exceptionnelle. Mais cette grande confiance dans les ressources de la philologie n'est cependant pas partagee par des auteurs comme Maxime Rodinson et Henri Charles (cf. Rodinson, Revue d'histoire des religions, 150, 1956, pp. 233-235 et Henri Charles, Mélanges de L'Université Saint-Joseph de Beyrouth, 32, 1955, p. 223).

$$
\text { I. LE SACRIFICE RELIGIEUX }
$$

I1 n'est pas possible, dans le cadre de cette note, de présenter 1 'ensemble des données connues -- aussi limitẻes et fragiles soientelles -- sur les cultes, pélerinages et pratiques sacrificielles de la jâhiziza (période de 1'ignorance, 1'anté-islam), bien qu'on ait souligné plus haut leur extrême importance pour une étude approfondie du sacrifice religieux. Les circonstances historiques dans lesquelles Mahomet a repris la coutume anté-islamique du pélerinage à la Mekke, en 1 'an 5 de 1 'Hégire d'abord avec l'expédition d'Hudaybiya, puis en 
$1^{\prime}$ an 7 où le Prophète accomplit lui-même les cérémonies rituelles à la Ka'aba, en l'an 8 où il délégua Abu Bakr comme chef de la caravane du pélerinage de 1 'adieu (hajjat $a$-wadốl'), ont étê relatées maintes fois par la Tradition (2). Le rituel suivi par le Prophète lors du pélerinage de $1^{\prime}$ adieu est, en principe, icentique à celui qui est pratiqué aujourd'hui et comportait donc le sacrifice de Mina, effectué le 10 ahul'hijja, le plus important des sacrifices religieux. Celui-ci apparaît donc directement repris de 1 'anté-islam, en ce qui concerne sa forme et son rituel (3), du moins, c'est donc au-delà de 1'islam qu'il faut aller chercher les origines du sacrifice religieux de Mina. Les données que l'on possède sur le sacrifice dans l'anté-islam sont cependant encore trop fragiles pour permettre une recherche approfondie sur ce point. Mais certains aspects du sacrifice actuel posent des questions qu'il est intëressant de rësumer ici :

1) En dépit d'une pratique très fréquente et d'un rôle très important, vraisemblablement (4), dans I'anté-islam, le sacrifice chez les Arabes $n^{\prime}$ apparaît pas fondé sur un mythe qui le comnente et l'explique, come c'est le cas dans certaines religions africaines. En effet, jusqu'ici, aussi loin que l'on puisse remonter dans l'histoire de l'Arabie

(2). Pour une analyse des motifs qui ont poussé Mahomet à reprendre la coutume anté-islamique du pélerinage à la Mekke, voir SnouckHurgronje, Het Mekkaansche Feest, Leyde, 1880, et, plus généralement, Encyclopédie istomique, Hadjdj, p. 33 sqq., Lème éd.

(3). A la veille de l'Hégire, les lieux de culte étaient très nombreux : points d'eau, haltes caravanières, lieux de foire, etc. Mais en Arabie occidentale, la Mekke était déjà un lieu de culte très important et la Ka'aba, cube de pierre posé sur une assise, semble, d'après la Géographìe de Ptolémée et certains récits légendaires, avoir été un sanctuaire déjà très fréquenté. Pour une étude des cultes et rituels anté-islamiques à la Mekke, cf. Snouck-Hurgronje, op. cit. et Encyclopédie islamique, hadjdj et Ka'aba. Le sacrifice de Mina du 10 dhul' hijja ou yawm al-adahi, "jour des sacrifices", constituait, semble-t-il, un des principaux éléments du rituel anté-islamique.

(4) L'attachement populaire, encore aujourd'hui, aux pratiques sacrificielles (voir supra, II. Le sacrifice populaire), en dépit des condamnations de l'islam officiel, constitue une indication précieuse sur 1'importance de celles-ci dans 1'antê-islam. 
occidentale, on constate l'absence de rêcits mythiques. Il semblerait que cette forme de pensée n'existait pas chez les Arabes du Hejaz avant l'islam. On explique gênéralement ce phênomène de deux manières :

-- la mentalité arabe, éminemment pratique, le genre de vie nomade auraient été peu favorables aux spéculations sur la nature et l'activité des puissances divines. Le "merveilleux" du bêdouin arabe se limiterait à $I^{\prime}$ humain (comme en témoignerait l'activité des poètes), ses héros également. Cette "explication" a êté consolidée par les témoignages des ethnologues et des voyageurs qui se sont accordés à constater l'irréligiosité du bédouin, sa profonde insouciance à l'égard de l'au-delà et son indifférence vis-à-vis du sacré.

-- La réaction musulmane aurait détruit délibérément toute trace de récit mythique concernant 1a jâhilizya.

Pour Joseph Chelhod, qui a consacré une étude spécialisée (L'Homme, 1962 ) et un chapitre de son Introduction à la sociologie de l'istam (Paris, 1958) à cette question, les deux explications seraient valables. Il estime, en effet, difficilement pensable que les villes, où le culte des idoles était plus actif que dans le désert, n'aient pas produit de

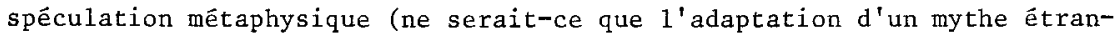
ger, puisque la Tradition veut que les idoles aient été importées. Pourquoi alors n'avoir pas importé le mythe avec les idoles ?). Là, il faudrait donc admettre l'intervention de la réaction islamique. J. Chelhod le fait d'autant plus facilement qu'il s'est efforcé (op. cit.) de montrer qu'il existe bien cependant un monde mythique (5) chez les Arabes bédouins, mais qu'en raison de la conception diffuse de la divinité qui aurait été alors la leur, (selon cet auteur) il serait vain de rechercher chez eux la forme classique du mythe. Dans le mythe arabe, les dieux seraient remplacés par un hêros civilisateur (humain), mieux encore par 1'ancêtre éponyme et les jinn, forces chtoniennes, y figureraient les êtres fabuleux. Ce mythe arabe aurait eu pour fonction de justifier certains phénomènes inexplicables de la nature, de motiver des pratiques rituelles et de fonder 1 'origine de certaines institutions ou de certains groupements chameliers, mais en se référant toujours à la vie quotidienne et

(5) Toufic Fahd (La divination arabe, Paris, 1966) est également convaincu qu'il y aurait eu "une pensée mythique latente" chez les anciens Arabes et que celle-ci est repérable dans plusieurs légendes à caractère onomastiquue (p. 15). Le vieux fond bédouin aurait êté caractérisé par des légendes topographiques très pauvres. 
en expliquant par analogie avec celle-ci. Or, il est possible, estime encore J. Chelhod (Introduction..., p. 134) de retrouver ces caractéristiques de la pensée mythique arabe ancienne dans la stricte orthodoxie musulmane. La littérature hagiographique et l'exégèse indiquent que les rêcits à tendance mythique jalonnent 1 'existence du Prophète. Les jinn sont toujours là et le héros civilisateur, incarné par la figure biblique d'Abraham, cautionne les nouvelles institutions. Les réfërences à Abraham sont constantes dans le Coran, c'est lui qui serait le véritable fondateur de $1^{\prime}$ islam. Selon ses propres dires, Mahomet n'a pas apportë de religion nouvelle, il est seulement l'"avertisseur" (nâahir), venu pour condamner le polythéisme et exhorter les hommes à embrasser la religion d'Abraham. C'est Abraham, affirme le Coran, qui a reconstruit la Ka'aba, purifié la demeure d'Allah et institué les rites du pélerinage à la Mekke, le sacrifice et la lapidation (Coran, II, 125 et 127). Ainsi, en effectuant un sacrifice (mouton, en particulier) à la Mekke lors du pélerinage, le musulman referait le geste d'Abraham, immolant une victime pour racheter son fils. Et, au-delà d'Abraham, la Tradition remonte jusqu'à Adam : 1e rite du sacrifice serait encore plus ancien et le bêlier d'Abraham serait aussi le mouton offert par Abel, agrẻe par Dieu et mis de côté pour servir de substitut à Isaac ou à Ismaill (Tabari, Annales). Croyances arabes ou emprunts au judaissme? Sans nier les influences judaíques, J. Chelhod voit dans cette continuité de tradition une conception cyclo-mythique de 1 'histoire qui, selon lui, aurait êté présente chez les Arabes dans 1'anté-islam et qui s'est appuyée, dans l'islam, sur la notion de paradis perdu : le paradis, puis le déluge, puis Abraham -- la religion prêchée par Abraham, selon le Coran, n'était pas le judaïsme, mais le hanéfisme (Coran, II, 135, III, 67, 95), islam avant 1a lettre -- puis nouvelle perte de foi et arrivêe de Mahomet, l'"avertisseur" et nouveau dêpart donc avec l'islam. Là, on toucherait, selon J. Chelhod, à une idée courante dans l'islam : décadence du présent par rapport aux moments historiques précédents. Mais nullement un éternel recommencement : à partir de Mahomet, la déchéance progressive ne sera pas suivie d'une nouvelle régénération. Son point extrême marquera seulement l'imminence du Jugement Dernier.

2) La place du sacrifice dans 1'expérience religieuse musulmane. Alors qu'il est pratique courante dans la vie quotidienne bédouine 
(cf. ci-dessous, II. Le sacrifice populaire), le sacrifice n'occupe pas une place centrale dans l'expérience religieuse musulmane. I1 n'y a pas identification entre le sacrifiant et sa victime (6). En outre, le sacrifice n'est pas obligatoire, mais seulement recommandé par le Coran et il admet des substitutions et des équivalences (jeûne et aumônes). Ces particularités ont amené certains auteurs (7) à considérer qu'il n'y a pas, dans l'islam, de sacrifice proprement religieux. Les sacrifices recommandés par le Coran, dans la mesure où ils sont de simples actes de dévotion et de piété, n'ont aucur lien avec la notion d'expiation. Lorsque cette notion intervient dans la pratique sacrificielle musulmane, elle est en effet totalement indépendante du sang versé et n'est fonction que du repentir du pêcheur et de la misêricorde divine. A 1'appui de cette thèse, le verset 37 de la Sourate XXII (Le pélerinage) : "Ni leurs chairs ni leur sang n'atteindra Allah, mais (seule) la piêté (venue) de vous l'atteindra. Ainsi (ces victimes) vous ont êté livrêes". (Trad. Blachēre). Mais d'autres (8), comme J. Chelhod (Le sacrifice chez les Arabes, Paris, 1955, pp. 57-60), s'appuyant sur des commentaires hanbalites (Ibn Qudama, Al-mughni was-shahr al-kabir, Le Caire, 1348), sur AI-Shâfi'i (Kitẩb al-'Umm, Le Caire, 1321), et des

(6). Pour les mystiques musulmans et notamment al-Hallâj, les rites intermédiaires entre I'Homme et Dieu sont caducs et la seule offrande véritable est celle de soi-même à Dieu. "Ils apportent des agneaux à sacrifier, moi $j$ 'apporte mes veines et mon sang", proclame-t-il. Cf. L. Massignon, La passion d'al-Hosayn Ion Manswo at-Halzâj, martyr mystique de l'istom (Paris, 1922).

(7). Cf. notamment :Encyclopédie Hastings (XI, p. 29) ; Loisy, Essai historique sur le sacrifice (Paris, 1920) : Massignon, E1 Hallaj, mystique de $1^{\prime}$ islam, Bulletin des études arabes, 43 (1949) ; G. H. Bousquet, Les grandes pratiques mituelles de l'islam (Paris, 1949); Doutté, Magie et religion dans Z'Afrique du Nord (Alger, 1909).

(8) Le débat sur cette question a été maintes fois repris et notamment dans un article récent du $\mathrm{R}$. P. Henninger (Deux études récentes sur 1'Arabie pré-islamique, Anthropos, 58, 1963), où s'affrontent deux sêries de donnêes, suggêrant des réponses opposêes :

a) l'intention du Prophète d'abolir les sacrifices de la jahiliya, tout en conservant les rites auxquels lui-même et ses partisans ont participé dans la première période mekkoise avec les incroyants, mais en "détachant, selon les termes de Gaudefroy-Demombynes, leur pensée des faux dieux et en la consacrant toute entière à Allah" (Mahomet, Paris, 1957, p. 92), c'est-à-dire en en changeant la signification profonde, le sens, en le dénaturant en fait de sa nature de sacrifice dans 1 'acception anté-islamique (le verset 37 de la Sourate XXII semble 
textes de Ghazâlî ('Ihyôa 'uZum al-d̂̀n, Le Caire, 1278) et Ibn Rushd (Averroès, Kitẩb bidâyat al-mujtahid wa nihâyat al-muqtasid, Le Caire, 1335) soutiennent que la croyance en l'efficacité du sang avec effet proportionnel à la quantité versée, est bien présente dans le sacrifice musulman. La persistance et la fréquence du sacrifice populaire en serait une preuve supplémentaire. D'ailleurs, Malik et Abu Hanifa (Ibn Rushd, op. cit.) jugeaient le sacrifice obligatoire. Cette conviction conduit $J$. Chelhod à rapprocher les mécanismes du sacrifice sanglant chez les musulmans de ceux observês par Marcel Griaule chez les Dogon (Dieu d'eau, Paris, 1948, p. 262) et à élaborer une thẻorie sur la

bien exprimer cette intention). Selon le R. P. Henninger, le projet de Mahomet aurait été de laisser subsister les immolations liées au pélerinage mais d'y remplacer l'êlément cultuel par un élément purement éthique, de ne concéder au rite qu'une importance symbolique, subordonnée, et non une efficacité propre. Cette tendance a été suivie par ceux des penseurs musulmans qui expliquent les sacrifices liés au pëlerinage non pas comme un don fait à Dieu (que peut-on d'ailleurs donner à Dieu qui ne lui appartienne déjà ?), mais comme une cérémonie commémorative du sacrifice d'Abraham ou comme une aumône puisque la viande du sacrifice n'est pas mangée pour l'essentiel par le sacrifiant, mais offerte aux pauvres.

A ces arguments, cependant, Maxime Rodinson (Revue d'histoire des religions, 1956, p. 232) oppose le fait que, dans l'islam officiel, le sacrifice, "quoiqu'on en ait dit et malgré l'effort de Mahomet (parallèle à celui des prophètes d'Israël) pour le mettre au second rang ou en spiritualiser le sens" a conservé un grand rôle et que le sentiment populaire y est très attaché (même le wahhabisme n'a pu l'abolir).

b) Dans 1'islam populaire, ethnologues et voyageurs ont constaté que les sacrifices anciens subsistent presque inchangés, adressés à Dieu ou plus souvent à un intermédiaire, ancêtre ou saint. Ceci en contradiction absolue avec l'intention du Prophète et la thêologie musulmane officielle. Pour Maxime Rodinson (op. eit.) ce phénomène constitue une "revanche" du système ancien prë-islamique contre le nouveau système religieux organisé par Mahomet, systēme marqué par la volonté d'introduire un nouvel esprit dans une forme ancienne et non de renouveler systématiquement la forme et le fond, c'est-à-đire d'opérer une révolution. La fonction ancienne n'aurait ainsi pas été abolie malgré la volonté du réformateur, l'effort des théologiens, des mystiques et des rationalistes au cours des siècles. M. Rodinson déplore que cet aspect du passage de 1 'anté-islam à 1 'islam n'ait pas été davantage étudié. Pour le R. P. Henninger, le débat est liê aux options théoriques gênérales dont dépend la définition du sacrifice : si le sacrifice consiste en fait en une opération magique pour l'élimination du mal (et pas nécessairement d'une offense morale), il est vraisemblable, compte tenu de la vigueur d'une telle conception dans le sentiment populaire arabe, qu'elle ait subsisté, malgré l'effort de Mahomet, 
nature du sacrifice arabe et sa fonction.

3) Le sacrifice religieux musulman n'est ni collectif, ni public. Seul le sacrifiant est concerné dans ses rapports personnels avec Dieu. Le sacrifice de Mina, le 10 dhul'hijja n'est qu'une juxtaposition de sacrifices individuels.

4) Le sacrifice peut être accompli par n'importe quel membre de la communautē. La femme peut prendre une part active au sacrifice et même imnoler ses propres victimes. L'islam n'ayant pas de clergé, c'est I'offrant lui-même qui doit, en principe, sacrifier ses propres victimes. Dans la pratique, I'offrant peut se faire remplacer, si la besogne matérielle le dégoûte par trop. Dans ce cas, n'importe qui peut faire l'affaire, à condition qu'il s'agisse de quelqu'un -- un musulman libre, un esclave, un sourd, un mineur, un incirconcis, un chrétien, un juif, une fenme peuvent pareillement convenir -- en possession de toutes ses facultés mentales (seuls donc un fou ou un homme ivre sont exclus), ceci, sans doute, en raison de l'importance accordée dans l'islam à l'intention réfléchie ou niya. C'est souvent aujourd'hui un boucher qui accomplit matériellement le sacrifice. Mais l'offrant, en tout état de cause, est obligé d'assister à l'immolation de sa victime. Il doit, sinon aider le sacrificateur, du moins lui tenir la main ou aiguiser l'jnstrument du sacrifice (Bukhra-Qastalânî, Sahthh, VIII, Al-Shafi'i, etc.).

\section{I.1. La classification des sacrifices selon les docteurs de l'islom.}

I1 n'y a de sacrifice que sanglant. Les immolations sont désignées par le terme de dhabtha, commun à toutes les langues sêmitiques (Ahabaha : égorger). La victime doit être abattue rituellement (9),

jusque dans la pensée des docteurs. Mais si le sacrifice est "l'oblation d'un don en honmage à un être supérieur auquel on se soumet, cette notion, bien vivante dans 1 'islam populaire, est à exclure de l'islam officiel.

(9) Il en est de même d'ailleurs pour les nourritures qu'il s'agisse de boucherie ou de chasse. Pour qu'elles soient legalement consommables, elles doivent également être abattues rituellement et 1 'invocation rituelle "Au nom d'Allah, le bienfaiteur misêricordieux", ou dans sa formule abrégée, "Au nom d'Allah" (bismilzâh) doit être prononcée sur l'animal. 
c'est-à-dire égorgée et le sang répandu, faute de quoi elle sera illicite (Coran, V, 4 et VI, 147 et surtout Tradition).

Les docteurs de 1'islam distinguent, en principe, deux catégories de sacrifices :

-- ceux qui sont offerts en fonction d'un voeu, en réparation d'une infraction, ou par obligation morale.

-- ceux qui sont simplement des actes de dévotion.

Mais cette classification n'est pas rigoureuse, et il y a souvent divergence de vues entre les docteurs dans l'identification de tel ou tel sacrifice. Plus précise est la classification en fonction du temps et du lieu du sacrifice (cf. Tahtâwi : Hasiza 'alad-dorpil-Mukhtâr', 1282) :

-- sacrifices effectués dans un lieu et un temps déterminés : le sacrifice de Mina, le 10 dhul'hijja (Mekke) et ceux qui correspndent aux intentions (niya) de tomattu' et de qirân. (voir ci-dessous).

-- sacrifices effectués le 10 ăhul'hijja, n'importe où dans le monde musulman, en rêplique du sacrifice de Mina pour ceux qui n'accomplissent pas le pélerinage.

-- sacrifices offerts en réparation d'une infraction aux obligations de 1 'ihrồm. (sacralisation) et sacrifices désacralisatoires ('ihsôcr ), obligatoirement effectués à la Mekke, mais à un moment variable.

-- sacrifices votifs n'importe où, n'importe quand.

A. Le sacmifice de Mina, le 10 dhul'hijja. Le plus important des sacrifices musulmans est celui qui est effectué à Mina, à la Mekke, le $10 \mathrm{dhul'hijja,} \mathrm{à} \mathrm{l'occasion} \mathrm{du} \mathrm{pélerinage.} \mathrm{La} \mathrm{victime} \mathrm{immolêe} \mathrm{porte}$ alors le nom de hady. Ce terme qui signifie "oblation" dérive de la racine arabe $\mathrm{h} d \mathrm{y}$, "guider", "mettre sur 1a bonne voie", mais aussi "faire un présent" (Encyclopédie islamique, nvelle éd., p. 55). C'est ce dernier sens que les lexicographes arabes ont retenu. Dans le Coran, le mot hady désigne des oblations destinées à la Ka'aba (II, 196, V, 2, 95, 96, XLVIII, 25, XXII, 33).

L'offrande d'un hady est, thêoriquement, facultative. Mais les prescriptions du hajj (pélerinage) en font une quasi obligation pour tout musulman libre en mesure d'acheter une victime.

Il faut rappeler $i c i$ que le pélerinage peut être accompli selon 
trois modalités, correspondant à trois intentions (niza) :

-- 1'ifrâa, ou "séparation", qui consiste pour le pélerin à faire le hajj seul, à l'époque prescrite, la 'umra, ou "petit pélerinage", qui primitivement avait lieu au mois de rajab d'après les traditions prêislamiques, devant être fait à une autre époque ou négligê (une 'umra pendant le temps du hajj était alors une chose inconcevable dont Mahomet (10) fut 1'initiateur). Dans ce cas, le fidèle n'est - en principe -pas obligé d'offrir un hady.

-- le tamattu', ou utilisation de la 'umra pour le hajj (Coran II, 196). Cette modalité consiste à accomplir la 'umpa à 1'époque du pélerinage, à se sacraliser (ihrôn) une première fois pour la 'umra, puis à reprendre la vie profane (ihlâl) pour se sacraliser à nouveau pour le hajj. L'état de sacralisation (ihrôm), impliquant le respect d'interdits (rapports sexuels, soins de toilette, chasse et, d'une façon gênérale, verser le sang et cueillir des plantes), le tomattu' dispense en fait d'une continence pénible et du respect de ces interdits dans l'intervalle entre la 'umra et le hajj. Cette facilité n'est cependant pas recommandable et "à quiconque fera usage de la 'umra jusqu'au pélerinage incombera ce qu'il (lui) sera aisé (de sacrifier) comme offrande (hady). (Mais) quiconque ne trouvera pas (à sacrifier se libèrera) par un jê̂ne de trois jours durant le pélerinage et sept jours lors de son retour, (soit) dix jours entiers" (Coran, II, 196).

-- le qirân, ou "union" qui consiste à prenare l'ihrôn à la fois pour le 'umra et pour le hajj et à ne se désacraliser qu'une fois le pélerinage terminé. Cette formule qui permet de faire toutes ses obligations religieuses $d$ ! un seul coup, prive cependant le qarin d'un surcroît de mérite. Elle est également considérée par les docteurs comme une facilité et exige d'être rachetée par une oblation, comme le tamattu'.

Les quatre madhâhib ont bien évidemment des opinions différentes sur le degré de mérite à attribuer à chacune de ces niya, donc sur l'importance des compensations dues. Dans tous les cas, cependant, il est spécifié que l'offrande d'un sacrifice (hady) peut être remplacée par le jeûne et l'aumône. p. $86 s q q$.

(10) Voir à ce propos Snouck-Hurgronje, Het Mekkaansche Feest, 
A.1. La victime offerte en hady. Elle doit satisfaire à certaines exigences d'âge et de beauté qui sont définies par le fiqh. L'âge lêgal varie selon les espèces : cinq ans pour les chameaux, deux ans pour les bovins, un an pour les caprins, six mois pour les ovins. (Ibn Qudâma, Sharh, et Ibn Rushd, Bidâya). En outre, la victime doit être grasse et sans dêfauts -- en particulier, elle ne doit ni boiter, ni être aveugle ou borgne, ni galeuse ni chétive, ni présenter de scarification rappelant le wasm anté-islamique (marque tribale constituant un titre de propriétê). Il semble que les femelles (11) doivent être prêférées aux mâles (Tahtawi, op. cit.), sauf en ce qui concerne le bélier (cf. GaudefroyDemombynes, Félerinage à la Mekke, Paris, 1923, p. 279), ce qui serait soit une "survivance païenne", soit une influence de la légence d'Abraham. Une victime de couleur blanche est préférée. En ce qui concerne le choix de l'espèce, les auteurs musulmans (notamment Ibn Qudâma, Ibn Rushd, Tahtawi) s'accordent à considérer que plus 1 'animal est grand, plus sa valeur sacrificielle est grande (selon J. Chelhod, la valeur de la victime est fonction de la quantité plus ou moins importante de sang qu'elle contient, cf. Sacrifice chez les Arabes, op. cit., p. 173). C'est ainsi qu'une victime choisie dans le petit bétail convient pour une personne tandis qu'un chameau ou un bovin peut rassembler de sept à dix personnes.

A. 2. Consécration du hady. Ni l'intention refléchie, niya, ni la désignation verbale ne suffisent à consacrer une victime hady. La consécration définitive et sans possibilitê de retour se fait par deux gestes rituels : le taqzîd et 1'ish'ar (12). Le taqzîd (Bukhari-Ansari, a1Shafi'i, Ibn Qudâma) consiste à suspendre au cou de l'animal une ou deux sandales (na'l) ou un morceau de peau au moyen d'un lien qui peut être une courroie de cuir, un sac, etc. Les docteurs ne sont pas d'accord sur le nombre des sandales : pour al-Shafi'i, il en faut deux, pour Malik, une seule suffit. Le hady une fois consommé, on doit faire l'aumône du na'z. L'ish'ar forme un rite coordonné avec le taqlîd (les deux rites

(11) Dans le cas d'une femelle, si celle-ci met bas, le petit doit être sacrifié en même temps que la mère.

(12) Pour une étude de l'origine et de la signification de ces rites, voir J. Chelhod, Le svmbolisme des sandales dans le rituel arabe, Anthropos ; Gaudef roy-Demombynes, Pélerinage à la Mekke, op. cit. 
sont nécessaires pour que la consécration soit complète). Il consiste à faire une marque à l'animal à bosse, c'est-à-dire chameau ou bovin à bosse. Quand 1'animal n'en a pas (chèvres et moutons) certains auteurs admettent que 1 'on procède à un simulacre de marquage. Le marquage est une entaille pratiquée avec un fer de lance, que l'on enfonce jusqu'au sang, puis on tache $1^{\prime}$ animal avec le sang (Bukhari-Ansari, op. cit.). Lorsque la consëcration est terminée, l'animal ne peut plus être échangé, vendu, remplacé ou hérité. Il peut cependant être monté par le sacrifiant si cela ne l'endommage en rien, car il doit être remis tout à fait intact à Allah.

Toute une jurisprudence existe -- variable selon les écoles et même les juristes -- pour les cas où 1 'animal choisi ne serait pas conforme aux exigences légales (âge, etc.), où il subirait un dommage ou blessure après la consécration, au cas où il serait volé, etc.

A. 3. La mise à mort. Le 10 dhul'hijja est appelé youm al-nahr, jour du sacrifice, ou 'id al-adhâ, fête du sacrifice, ou encore 'iá $a \imath-k a b \hat{\imath} r$, grande fête. C'est ce jour-là que les pélerins sacrifient dans la vallée de Mina, près de la Mekke. Tout Mina, selon les docteurs, est lieu d'immolation et il n'y a pas, en principe, de lieu d'abattage fixé par la loi. Mais les immolations ont lieu, de préférence, à l'extrémité occidentale de la vallêe (Burckhardt, Travels, II, Burton, $A$ Pilgrimmage, II). Les pratiques rituelles recommandées au sacrifiant sont : 1) réciter la bismizlâh ; 2) faire la salât 'ala l'nab̂, la bénédiction sur le Prophète ; 3) se tourner vers la qibla, la niche qui marque pour les fidèles la direction de la prière ; 4) prononcer un triple takbir (la formule "Allah est très grand") ; 5) demander que le sacrifice soit accepté avec bienveillance (cf. Encyclopédie istomique, nvelle éd., p. 1033).

L'abattage rituel peut alors avoir lieu. On procède à l'égorgement, le ahaka'a, selon la méthode du ahabh ou du nahr, faute de quoi la bête serait considérée comme charogne (mayta), donc rituellement inconsommable (cf. Encyclopédie istamique, nvelle êd., p. 220). Au moment de l'abattage, il est obligatoire d'en formuler l'intention et d'invoquer le nom d'Allah. La victime est égorgée, la tête dirigée vers la qỉbla, couchêe sur le côté gauche si elle est d'espèce bovine ou ovine, debout entravée, sauf de la patte inférieure droite, si c'est un chameau. Dans le cas d'un chameau, l'égorgement rituel est le nahr, c'est-à-dire un 
coup de couteau dans le creux du sternum au-dessous de la gorge. Dans le cas des bovins et ovins, la bête a la gorge tranchêe (dhabh), y compris la trachée artère et l'oesophage. La tête n'est pas tranchée.

L'immolation doit se faire en un seul temps, sous peine de nullité.

A. 4. Instmuments de mise à mort. Il existe des discussions casuistiques sur la nature de l'instrument à employer. Le couteau, en tout cas, doit être très bien aiguisé et tout doit être fait pour épargner les souffrances de la victime.

A. 5. Consommation de la vionde du sacrifice. Les pélerins sacrifiants consomment une partie de la viande (le tiers, en principe) et distribuent le reste aux pauvres à titre de sadaqa, aumône. Ce que les pauvres ne mangent pas reste étalé au soleil.

B. Les sacrifices du 10 dhul'hijja, n'importe où dons le monde musulman. Au moment où, à Mina, ont lieu les sacrifices du yocum al-nahr, partout ailleurs dans le monde musulman, les fidèles, à l'instar des pélerins, sacrifient aussi. Ces sacrifices du 'id al-kabîr, la grande fête, sont appelés dahaya (13) (sing. dahiya), du verbe dahhâa, immoler. Leur origine semble être anté-islamique. Des Traditions attestent, en tout cas, qu'ils êtaient pratiqués dès les dêbuts de la période hêgirienne et que le Prophète en aurait fait une sunna, une coutume, pour tous les musulmans libres, en mesure d'acheter une victime. Mais le sacrifice de la "grande fête" n'est un devoir d'obligation (wôajib) que s'il correspond à un voeu (nadhr), pour la plupart des auteurs musulmans, exception faite d'Abu Hanîfa qui considère la dahiya comme obligatoire pour tous.

La dahiya peut être considêrée comme une réplique du sacrifice de Mina, dans la mesure où elle doit avoir lieu à la même heure, dans la matinée du 10 dhul'hijjja, après la prière du matin. Ce temps peut être prolongé jusqu'au soir de la même journée et même aux deux journées suivantes, suivant la majoritê des auteurs musulmans. Pour al-Shafi'i

(13) Le Coran n'emploie jamais le terme de dahiya, courant cependant dans les recueils de hadâth et dans l'usage, mais toujours celui de hady. Pour une origine possible de l'emploi du mot dahiya, cf. J. Chelhod, Sacrifice chez Les Arabes, pp. 50-53. La plupart des ouvrages de fiqh s'accordent à employer le mot dahiya pour les sacrifices du $10 \mathrm{dhul} h i j j$ ja effectués ailleurs qu'à la Mekke, réservant le terme de hady au sacrifice de Mina. 
cependant, si la dahiya n'est pas accomplie dans les heures légales, entre la salat al'id et le coucher du soleil, elle devient une immolation ordinaire sans effet spécial.

La dahiya, comme le hady de Mina, implique pour le sacrifiant une sorte de sacralisation (ihrôn) partielle, selon certains auteurs, dont Ibn Rushd, mais les uns en font une simple recommandation, tandis que les autres y voient une obligation. Cette sacralisation consiste, pour le sacrifiant, dès le premier jour du mois de ahul'hijja, à ne plus toucher à ses cheveux ni à ses ongles.

Comme pour le hady, la bête choisie pour la dahiya sera une bête légale et sans dêfauts. Mais la consêcration en est beaucoup plus simple que pour le hady. Il suffit que le sacrifiant prononce la formule "ceci est une victime" pour que la consécration soit effectuée. Mais si la prononciation effective de cette formule est indispensable pour l'école hanbalite, elle ne l'est pas pour les écoles hanéfite et malékite, qui estiment que la niya, intention refléchie, suffit à la consêcration. Comme pour le hady, la consécration est sans retour : la victime doit être obligatoirement immolée et ne doit subir aucun dommage entre sa consécration et son immolation.

Les pratiques rituelles précédant la mise à mort, de même que les techniques d'abattage rituel, sont semblables pour la dahiya et le hady. En ce qui concerne la viande sacrificielle, il est d'usage, come pour le hady, que le sacrifiant en consonme une partie (le tiers en principe) et distribue le reste aux pauvres. Mais sur ce point encore, il y a divergences de vues entre les docteurs et la part réservée au sacrifiant peut aller d'une simple bouchée jusqu'à la quasi-totalité de la dahiya. Cependant, si la dahiya correspond à un voeu, le sacrifiant n'en doit rien manger et doit distribuer la totalité de la viande (cf. Encyclopédie iszamique, nvelle éd., p. 1033).

C. Les sacrifices accomplis à la Mekke sans obligation précise de date. Ce sont les sacrifices qui viemnent racheter, le cas échéant, les infractions commises par le pélerin aux interdits et obligations de 1 'ètat d'ihrồn.

Consécration temporaire du pélerin à Allah, l'ihrâm doit être respecté, sinon celui-ci encourt la colère divine et risque le châtiment. Il doit donc racheter les infractions qu'il commet éventuellement pen- 
dant qu'il est en état de sacralisation -- s'il veut que son hajj soit agréé par Allah -- soit par un sacrifice, soit par le jeûne ou l'aumône. Mais 1'un et 1'autre doivent être accomplis à la Mekke même, dans le sanctuaire, sans condition de temps toutefois. Le pélerin peut se racheter à n'importe quel moment de son séjour dans la ville sainte.

I1 y a deux grandes catégories d'infractions :

-- Les empêchements matériels divers pour le pélerin de se rendre à la Ka'aba après s'être sacralisé. Le Coran (II, 196) dit : "Si vous êtes empêchẻs (1ibérez-vous) parce qu'il vous sera aisê (de sacrifier) comme offrande (hady). Ne vous rasez point la tête avant que l'offrande (hady) ait atteint le lieu de son immolation." 'uhsirtum, "si vous êtes empêchês", implique, selon R. Blachère, un empêchement survenant au cours de $I^{\prime}$ accomplissement du pêlerinage et sans doute, à l'origine, réfèrerait à un empêchement subi par les croyants de la part des polythêistes mekkois. Par la suite, les écoles juridiques ont joué sur 1 'ambiguité du terme et ont appliqué cette disposition au croyant qui, résidant loin de la Mekke ou étant dans l'incapacité de faire le pélerinage, s'en acquittait par mandataire. D'autre part, la nature de 1 'empêchement auquel fait allusion le verbe 'ahsara a fait l'objet de nombreux commentaires. Le cas de "maladie" est clairement prévu par 1a suite du verset : "A quiconque parmi vous sera malade ou atteint d'un mal affectant la tête, incombera rachat par jeûne, une aumône (sadaqa) ou un sacrifice rituel (nusuk)." Aussi des juristes tels al-Shafi'i y voient-ils une allusion à la situation du Prophète à Hudaybiya.

J. Chelhod voit dans le sacrifice ainsi demandé un sacrifice dẻsacralisatoire (Sacrifice chez tes Arabes, pp. 85-86), mais les divergences entre les écoles sur le caractère obligatoire de ce sacrifice, de même que sur le temps et le lieu de cette immolation éventuelle, ne permettent pas de conclure (pour Malik, le sacrifice est superflu, pour Abu Hanifa et al-Shafi'i, il est indispensable, mais pour le premier, il doit avoir lieu à la Mekke. en dehors du 10 dhul'hijja cependant et pour le second, dans le cas où le sacrifiant est assiégé, il peut y avoir immolation, sur place, hors de la Mekke, mais dans tous les autres cas 1'immolation doit avoir lieu à la Mekke (14).

(14) Ces divergences ont été souvent expliquées par la situation historique des débuts de l'islam : l'hostilité des Quraych à Mahomet rendait difficile pour les musulmans l'accès de la $\mathrm{Ka}$ 'aba avec les animaux du sacrifice. Voir à ce sujet, J. Chelhod, op. cit. p. 86 
-- Les manquements aux interdits et obligations de l'ihrồm.

- Violation de l'interdit de chasse. "Ne considérez point comme licite le gibier (tuê) alors que vous êtes sacralisé" (V, 96). Le pélerin doit donc observer cet interdit tant que dure son ihrôm, et non pas seulement à la Mekke dans le sanctuaire. Mais pour qu'il y ait délit, il faut que l'acte ait été délibérément accompli et nón pas par inadvertance. La rupture de l'interdit de chasse exige une compensation : "Quiconque parmi vous en tuera intentionnellement (devra ou bien) une compensation égale à la bête de troupeau qu'il tue en offrande consacrée à la Ka'aba -- deux hommes intègres parmi vous en jugeront -- ou bien son rachat sera la nourriture d'un pauvre, ou bien un jeûne équivalent à cela. (Tout cela est fait) pour que le (pécheur) goûte le châtiment de son geste." (Coran, V, 95).

- Violation de 1'interdit des rapports sexuels, rasage de la chevelure avant le sacrifice de Mina, manquement aux vêtements usuels de 1'ihrôn, sacralisation trop tardive (après avoir dépassé les frontières du harom, le territoire sacré), toutes ces infractions doivent être rachetées par 1'immolation d'une victime, selon la Tradition du moins, car le Coran ne précise rien.

Ici encore, les écoles divergent quant à 1'obligation du sacrifice pour chacune de ces infractions et encore davantage quant au lieu et au moment précis où ils doivent ëventuellement être effectués (toujours à la Mekke cependant). Elles s'accordent en revanche pour estimer que le sacrifice peut toujours être remplacé par le jeûne ou l'aumône et que le sacrifiant doit s'abstenir de consommer la viande, le sacrifice ayant un caractère réparateur.

D. Sacrifices votifs, n'importe où, n'importe quand. Ils sont affaire personnelle du sacrifiant et ne font pas l'objet d'une législation rigide comme celle qui marque les grands sacrifices officiels dont on vient de parler. Ils ne sont pas le résultat d'une infraction ou d'une violation d'interdit et s'ils constituent une obligation, c'est dans la:mesure oũ ils sont la conséquence d'une engagement librement consenti, d'une promesse faite à Allah par le sacrifiant. C'est donc le sacrifiant qui en fixe le lieu et 1e moment et qui choisit la victime en fonction de l'obligation qu'il a contracté. En principe, la viande d'un sacrifice votif doit être entièrement abandonnêe aux pauvres. 
I1 n'est pas question, dans le cadre limité de cette note, d'entrer dans le détail des multiples pratiques sacrificielles populaires. D'ailleurs l'ethnographie à laquelle un tel travail devrait faire appel est encore très incomplète ou insuffisamment dépouillée, selon les régions.

On n'abordera donc la pratique du sacrifice populaire que très brièvement et dans la mesure où elle peut contribuer à éclairer certaines des questions que soulève l'existence du sacrifice religieux, dans $1^{\prime}$ islam.

II. 1. Spécificités du sacrifice populaire arabe.

1) Son extrême fréquence. Il marque toutes les grandes étapes de la vie, de la naissance à la mort (naissance, sevrage, circoncision, engagement de mariage, mariage, consommation du mariage, installation des mariês dans leur tente, occupation d'une nouvelle tente, répudiation, mort), différents évênements tels que le partage du butin d'une razzia, une nouvelle récolte, une mise bas dans le troupeau, la naissance d'une pouliche, etc. Par ailleurs, des sacrifices sont effectués pour conjurer des malheurs, des calamités naturelles, épidémies, sécheresse, etc., par suite de multiples voeux et promesses, etc. Le sacrifice populaire est si fréquent que des observateurs comme A. Blunt (Pélerinage au Nedjed, bercecau de la race arabe, Paris, 1882) ont pu écrire qu'en sacrifiant ainsi en toutes occasions, les Arabes "ne croient pas obéir à un rite religieux, mais à une coutume" (p. 63).

2) La diffusion de ces pratiques (malgré une grande variêté selon les régions) dans tout 1 'ensemble du monde arabe, qu'il s'agisse de 1 'Orient ou du Maghreb.

3) Elles ne sont nulle part institutionnalisées et sont effectuêes le plus souvent individuellement, ou par un groupe tribal en tant que tel. Le choix de la victime est l'affaire du sacrifiant.

4) Elles s'adressent non pas directement à Allah, mais à des intermédiaires (saints) ou encore aux morts, aux ancêtres et même, parfois, 
aux jinn (cf. Musil, Jaussen, Doughty entre autres pour la péninsule arabique. Doutté. Massignon, Westermarck pour 1'Afrique du Nord). Pour cette raison, certains auteurs pensent que ces pratiques sacrificielles seraient seulement liêes aux conceptions bédouines de la vie et de la mort, à la notion de personne. Elles se situeraient ainsi dans la continuité directe de l'anté-islam (15).

5) Ces pratiques sont condamnëes par le Coran et la Sunna et cependant elles ont, à travers les siècles, résisté à ces condamnations et persisté dans leur. fréquence dans tout le monde arabe.

II. 2. Les différents sacrifices populaires.

La finalité des sacrifices populaires est toujours étroitement liêe à leur destinataire -- morts, ancêtres, saints ou ... jinn -et, selon les cas, ils visent seulement à apaiser ou bien à obtenir I'intercession de leur destinataire auprès d'Allah en faveur du sacrifiant.

1) Sacrifices aux morts. Il n'y a pas vêritablement de culte des morts dans les sociétés bẻdouines. Les observateurs ont même été frappés (Musi1, The Manners and Customs of the Rwala bedouins, New-York, 1928 ; Arabia deserta, New-York, 1927 ; et Lammens, Fatima et les filles de Mahomet, Rome, 1912 ; Jaussen, Coutumes des Arabes au pays de Moab, Paris, 1948, etc.) par le peu de cérémonie et la relative rapidité avec laquelle on procède aux funérailles. A. Musil observe chez les Rwala (pp. 670 sqq.) une crainte superstitieuse du cadavre, qu'on enterre couchê sur le dos, un bras repliê sous la tête et qu'on recouvre d'un tas de pierres pour le protéger des bêtes. Mais si la crainte pousse

(15) Dans la mesure où le Coran reflète les anciennes conceptions bédouines, il fournit certaines données sur la représentation de l'homme chez les Arabes anté-islamiques. Cependant, du fait que le Prophète avait non seulement à se démarquer des croyances de la jâhi izya, mais aussi des notions juives et chrétiennes, le Coran reste sur une prudente réserve (XVII, 85 sqq.). Pour une analyse des données contenues dans le Coran sur les deux principes vitaux, la nafs et le wh, dont I'homme est dotë, voir notamment $\mathrm{R}$. Blachère, Notes sur le substantif nafs... dans le Coron, Semitica, 1948. D'autres indications sont données par les recueils de hadith, notamment sur les croyances concernant le sort des principes vitaux après la mort. 
à se dêbarrasser ainsi rapidement du cadavre, on n'en procède pas moins, au moment du décès et de l'inhumation, à des libations et surtout à des sacrifices, souvent un chameau, plus rarement un cheval. Dans la croyande ces Rwala (p. 672), comme dans celle des Arabes de Moab (p. 353), la victime est destinée au mort et lui servira de monture dans I'au-delà. A cette fin, le rituel exige que chaque morceau de la viande sacrificielle soit passé au feu quelques instants, avant d'être consommé par tous les membres du groupe, surtout les pauvres. Ceux qui appartiennent à des lignages nobles (Rwala, p. 671) s'abstiennent au contraire de participer à ce repas. Chez les Arabes de Moab, on immole en outre une victime sous la tente du défunt (pp. 101 et 352).

Tous les observateurs s'accordent à attester la permanence de ces pratiques chez les Arabes (Doughty, Travels in Arabia Deserta, Cambridge, 1888 ; W. G. Palgrave, Une année de vayage dans l'Arabie centrale, Paris, 1866 ; Burckhardt, Travels..., 1829, entre autres), bien qu'elles soient formellement condamnées par 1'islam officiel (Coran, XXXI, 21, XXXIV, 43). Le sacrifice dû aux morts ne se limite pas au moment de l'inhumation, il doit être réitêré pẻriodiquement (jusqu'à la troisième génération, selon Doughty) si $1^{\prime}$ on ne veut pas provoquer la colère du défunt. Il s'agit done avant tout d'apaiser le mort et d'éviter les effets néfastes de son éventuelle colère.

2) Sacrifices aux ancêtres. Les ancêtres ne sont pas forcément les véritables fondateurs de tribus; de clans ou de lignages, mais plus souvent des défunts qui, de leur vivant, se sont distingués par leur bravoure, par des actions d'éclat ou ont acquis un grand prestige de quelque manière que ce soit (prodigalité par exemple). Leur sépulture est marquée par une tente ou par une qubba et lorsqu'on passe devant, on y dépose généralement une modeste offrande (cf. I. Goldziher,Culte des ancêtres, Revue d'histoire des religions, 10, 1884). Au cours des réunions tribales, les descendants de $I^{\prime}$ ancêtre procèdent en son honneur à de nombreuses immolations dont le rituel a été décrit par Jaussen (p. 316) pour les Arabes de Moab (16). Une pratique très répandue est

(16) "La chamelle ou les brebis sont amenées près du tombeau ; le cheikh, s'il s'agit d'un honneur à rendre par toute la tribu ou un simple particulier, s'il s'agit d'un honneur privé, s'empare de la victime, l'égorge en disant 'don et gratification à mon ancêtre un tel'. Le sang de la victime est recueilli dans un sag -- plaque en fer bombé sur laquelle les Arabes font cuire leurs galettas de pain -- pour oindre d'abord le tombeau, ensuite les chameaux, afin d'attirer la bénédiction..." 
le voeu d'immoler une victime à l'ancêtre pour s'assurer le succès d'une expédition guerrière, d'une razzia, etc. Le voeu est destiné à provoquer 1'intérêt de 1 'ancêtre pour la personne l'ayant formulé et le pousser à favoriser, par son intervention "supérieure", les entreprises de celle-ci. Dans ces cas, le sacrifice n'a pas nécessairement Iieu au tombeau de l'ancêtre, mais souvent au campement au retour de I'expédition (Jaussen, Moab, p. 305).

Le sacrifice aux ancêtres vise donc surtout à attirer les faveurs de ceux-ci et à les inciter à utiliser leur pouvoir dans 1'au-delà au service de leurs descendants. Les ancêtres ne font ainsi, semble-t-il, pas l'objet d'un véritable culte, mais d'une vénération occasionnelle à des fins précises.

3) Les sacrifices adressés aux saints. Le passage de l'état d'ancêtre à celui de saint est difficile à apprécier. D'une façon générale, est reconnu saint, wali, celui qui fait éclater après sa mort la preuve de la puissance qu'il détient de Dieu (baraka). Alors il devient l'objet d'une vénération à la mesure de son efficacité (protecteur, guérisseur, gardien des biens et des troupeaux, il répand la prospéritë sur la région, enrichit ses fidèles, etc.). Le wali est avant tout un personnage local (17) et son culte ne peut être dissocië, en général, de sa sépulture (les tombeaux des saints servent souvent d'asile pour les fugitifs, de lieu de pélerinage populaire; ils sont porteurs de la baraka du saint qui y est inhumé et il est fréquent de jurer sur une tombe). Aussi, alors qu'un sacrifice à un ancêtre peut être effectué au hasard des déplacements de la tribu, celui que l'on adresse au saint est effectué sur son tombeau. D'autre part, alors qu'un ancêtre agit, semble-t-il, de sa propre initiative, le wazi reçoit d'Allah la possibilité d'agir, c'est Allah qui lui donne la baraka, et son rôle est seulement celui d'un intermédiaire entre Allah et les hommes. Le tombeau d'un saint reconnu très efficace est un lieu de sacrifice fréquentē : les victimes y sont immolëes en grand nombre et leur sang gênêralement répandu sur le tombeau tandis que la viande est distribuée à I'assistance (Jaussen, Moab, pp. 309-310). Ces sacrifices ont pour

(17) En Libye comme au Soudan nilotique, on a constaté que les wali sont nombreux chez les pasteurs arabes bouviers. Les chameliers, en revanche, connaissent peu (ou pas, Musil, Rwala, p. 417) le culte des saints et la tombe sacrée. Leur existence semble donc plus ou moins liée à la présence de sédentaires ou semi-nomades. 
objet d'obtenir aide et assistance du saint et sont souvent associés à d'autres offrandes.

Le culte des saints jouit partout d'une grande ferveur populaire, mais l'islam officiel lui est très hostile et le considère comme tout à fait incompatible avec la Sunna.

4) Les sacrifices adressés aux jinn. En dépit de l'islam, nombre de sacrifices continuent à s'adresser aux jinn, les habitants de la terre, 'ahl al-'ard, créatures invisibles qui s'incarnent dans leurs manifestations à I'homme, dans toutes sortes d'apparences, humaines ou surtout animales (Jaussen, Musil, Doughty, etc.). Depuis I'anté-islam, ils sont nombreux à hanter les:ruines, les lieux déserts et sauvages, les points d'eau. Le Coran a reconnu leur existence (Sourate LXXII, Les djinns) et bien qu'il les divise (verset 14) en "Soumis à Allah (muslim) et en "Révoltés" (qâsit), la croyance populaire les considère nuisibles par nature, causant toutes sortes de maladies et de désastres, possêdant les déments (Musil, Rwala, p. 398). Mais habitants de 1a terre, 'ahl al-'ard, ils sont les maîtres des lieux qu'ils hantent et il est bon de les amadouer par un sacrifice (Jaussen, Moab, p. 319) lorsqu'il s'agit d'installer une nouvelle tente ou même d'agrandir une ancienne demeure, d'utiliser une nouvelle source, etc.

Les quelques données contenues dans cette note ne permettent bien évidemment pas une réflexion approfondie sur la nature et le rôle du sacrifice dans les cultures arabes musulmanes. On se bornera donc ici à un bref exposé des thèses qui ont étê développées, à partir de données relevant de ces cultures.

Sans fondement cosmogonique connu et cependant d'une fréquence dans la vie quotidienne qui a permis de dire qu'il était "une véritable panacée", repris par l'islam qui n'en fait cependant pas un élément central de l'expërience religieuse et admet que lui soit substitué jeûne et aumône, le sacrifice sanglant, chez les populations arabes musulmanes a été considéré, on l'a vu ci-dessus, par certains auteurs 
comme plus proche d'une assez vague coutume que d'un acte ayant des implications religieuses. Ainsi Raymond Firth (Encyclopaedia Britanni$c a$, "Sacrifice", p. 135) écrit : "Faint shadows of sacrifice as it was practiced by the pre-islamic Arabs have influenced Muslims, so that they consider every slaughter of an animal an act of religion. They also celebrate feasts in fulfillment of a vow or in thanksgiving for good fortune, but there is no sacrificial ritual connected with these festive meals. On the last day of the annual Pilgrimmage to Mecca, animals are sacrificed; nevertheless, it is not the sacrificial rite that is important to the Muslims, but rather their visit to the sacred city."

La thèse qu'avait soutenue $W$. Robertson Smith (Lectures on the retigion of the Semites, Londres, 1927), à partir de donnees concernant les bédouins nomades est aujourd'hui abandonnée. Elle se fondait sur 1 'hypothèse du totémisme dans les anciennes sociêtês arabes. Le sacrifice sanglant, qu'il analysait à partir du récit du sacrifice d'un chameau blanc par les Sarrasins au IVe siècle, serait à l'origine chez les Arabes un repas communiel à caractère totémique. Et il s'agissait 1à, selon cet auteur, de la forme la plus primitive du rite.

La thèse de Joseph Chelhod (Sacrifice chez les Arabes, Paris, 1955, pp. 181-198), beaucoup plus récente, a été largement influencée par les travaux de Marcel Griaule : le sacrifice arabe serait un moyen d'approche du divin, fondé sur le don et le pouvoir rédempteur du sang. S'il y a bien communion, celle-ci serait provoquée par le don et non pas par le repas sacrificiel. Le sacrifice aurait pour fonction "non seulement de lier et purifier, mais aussi de rompre l'équilibre, de parfaire le mouvement cyclique de l'énergie créatrice... D'où cette fréquence des immolations dans la vie civile. Chaque événement important, chaque étape nouvelle de la vie est un pas dans 1 'inconnu et entraîne un détournement illicite de l'énergie, un heurt prémédité du sacré. Il dèvient urgent d'en détourner les conséquences, d'en prévenir les menaces. Une force de même nature est donc dêpêchée dans le but précis de faire un contre-poids au dêséquilibre provoqué, voire de créer un désêquilibre qui amène de la part de l'énergie créatrice, un abandon de forces en vue de rétablir 1'ordre normal des choses." (p. 199). Cette thèse, qui s'appuie sur les thêories générales de $1^{\prime}$ auteur concernant le développement de la religion (à l'origine, il y aurait 
eu un "sacrê diffus", religion et magie auraient été confondues dans l'esprit des hommes et ne se seraient diffërenciées que progressivement), amène J. Chelhod à voir dans les sacrifices religieux des sacrifices expiatoires à 1'origine et non de simples offrandes comme l'affirment les docteurs de 1'islam. Soulignant d'une part les contradictions qui marquent les positions des quatre madhâhib et d'autre part insistant sur la volonté de réaction contre les pratiques anté-islamiques que 1 'on trouve dans le Coran (le fameux verset XXII, 37, đêjà cité, en est pour lui une illustration), cet auteur affirme qu'en autorisant expressêment le sacrifiant à manger sa victime (XXII, 28 et 36), le Prophète a surtout voulu abolir la coutume anté-islamique qui aurait fait de la victime du sacrifice la nourriture des dieux. Et les penseurs de 1 'islam auraient négligé de prendre en compte dans leurs interprétations, le problème de la souillure déchargée sur la victime dans le cas d'une expiation, souillure qui la rendait impropre à être consommée par le sacrifiant et consommable seulement par les dieux. Aussi, le fait que le Coran autorise le sacrifiant à consommer la victime du sacrifice ne suffit pas à prouver, pour J. Chelhod, que le sacrifice n'est pas expiatoire et il tente'de montrer, à partir d'une êtude minutieuse des pratiques de la consécration du hady de Mina et également de celles qui sont liées au tomattu' et au qirôn et qui donnent lieu à des sacrifices de rachat, que le hady est en fait un sacrifice expiatoire : il viserait à purifier le pélerin de ses souillures et lui permettrait d'entrer en communion avec le sacré. Ce qui confirmerait aussi la présence dans $I^{\prime} i s l a m$ des nombreux sacrifices de rachat en cas de violation d'interdit.

Mais le R. P. Joseph Henninger (op. cit. pp. 462-464) estime que $J$. Chelhod fait trop de cas du pouvoir magique du sang, pouvoir sur lequel serait basé la rédemption. Cette erreur aurait pour origine une distinction insuffisament nette entre magie et religion. D'autre part, il ne lui parâ̂t pas prouvê qu'il existe bien des sacrifices expiatoires dans 1'islam populaire aujourd'hui et encore moins qu'il en ait existê dans l'anté-islam. Pour lui, ce que J. Chelhod qualifie d'expiatoire serait un rite d'élimination et pourrait être plus justement qualifié de sacrifice de rachat, le rachat pouvant concerner aussi bien un mal physique qu'un manquement à une règle et non pas la réparation d'une offense morale. M. Rodinson va dans ce sens aussi lorsqu'il écrit : 
"Nous en tenant au sentiment populaire ... il n'est pas sûr que la notion d'expiation y soit exclusive. Il s'y mêle la demande, l'action de grâce, la croyance à la possibilitê par le sacrifice d'écarter un mal de sa vie sans pour cela qu'on se demande si celui-ci est causé par une offense ou non" (Revue d'histoire des religions, 150, 1956). La souillure déchargée sur la victime s'explique suffisamment par l'élimination d'un mal physique et n'exige pas l'intervention d'une offense morale. Certes l'islam a ajouté la conscience d'une offense morale à expier, mais pour le R. P. Henninger, il n'est pas certain que cette notion ait franchi la pensée théologique et qu'elle soit comprise dans les croyances populaires. 